\title{
Multi-morbidities are Not a Driving Factor for an Increase of COPD-Related 30-Day Readmission Risk
}

\author{
Shuo-yu Lin (D) \\ Hong Xue \\ Yangyang Deng \\ Askar Chukmaitov
}

Department of Health Behavior and Policy, School of Medicine, Virginia Commonwealth University, Richmond, VA, USA
Correspondence: Hong Xue; Askar Chukmaitov

Department of Health Behavior and Policy, School of Medicine, Virginia

Commonwealth University, One Capitol Square, 830 East Main Street, Fourth

Floor, Richmond, VA 23219, USA

Tel + I 804-628-7529; + I $804628-3398$

Fax + I 804 628-1223

Email hong.xue@vcuhealth.org;

askar.chukmaitov@vcuhealth.org
This article was published in the following Dove Press journal: International Journal of Chronic Obstructive Pulmonary Disease

Background and Objective: Chronic obstructive pulmonary disease (COPD) is the third leading cause of death in the United States. COPD is expensive to treat, whereas the quality of care is difficult to evaluate due to the high prevalence of multi-morbidity among COPD patients. In the US, the Hospital Readmissions Reduction Program (HRRP) was initiated by the Centers for Medicare and Medicaid Services to penalize hospitals for excessive 30-day readmission rates for six diseases, including COPD. This study examines the difference in 30-day readmission risk between COPD patients with and without comorbidities.

Methods: In this retrospective cohort study, we used Cox regression to estimate the hazard ratio of 30-day readmission rates for COPD patients who had no comorbidity and those who had one, two or three, or four or more comorbidities. We controlled for individual, hospital and geographic factors. Data came from three sources: Healthcare Cost and Utilization Project (HCUP) State Inpatient Databases (SID), Area Health Resources Files (AHRF) and the American Hospital Association's (AHA's) annual survey database for the year of 2013. Results: COPD patients with comorbidities were less likely to be readmitted within 30 days relative to patients without comorbidities (aHR from 0.84 to $0.87, \mathrm{p}<0.05$ ). In a stratified analysis, female patients with one comorbidity had a lower risk of 30-day readmission compared to female patients without comorbidity $(\mathrm{aHR}=0.80, \mathrm{p}<0.05)$. Patients with public insurance who had comorbidities were less likely to be readmitted within 30 days in comparison with those who had no comorbidity (aHR from 0.79 to $0.84, \mathrm{p}<0.05$ ).

Conclusion: COPD patients with comorbidities had a lower risk of 30-day readmission compared with patients without comorbidity. Future research could use a different study design to identify the effectiveness of the HRRP.

Keywords: multi-morbidities, readmission, gender difference, health insurance, all-payer claims databases

\section{Introduction}

Chronic Obstructive Pulmonary Disease (COPD), according to World Health Organization (WHO) and Global Initiative for Chronic Obstructive Lung Disease (GOLD), is a lung disease characterized by persistent chronic obstruction of lung airflow that interferes with normal breathing and/or alveolar abnormalities usually caused by significant exposure to noxious particulars or gases. ${ }^{1,2}$ In 2011 , COPD was the third leading cause of death in the United States, and the second leading cause of reduced disability-adjusted life years (DALYs), trailing only ischemic heart disease. ${ }^{3,4}$ Moreover, the total costs of COPD treatment are projected to 
increase from $\$ 32.1$ billion in 2010 to $\$ 49.9$ billion by 2020 , mostly due to the increase of disease severity, hospital stays, and more complex multimorbidity. ${ }^{5,6}$ Multimorbidity, defined as patients having two or more coexisting diseases, is one of the key reasons for rising health care costs and a loss of productivity in the United States. ${ }^{7}$ The majority of COPD patients have comorbidities: $68 \%$ had at least one, and $16 \%$ had two or more, while $30 \%$ of those admitted with COPD acute exacerbation had four or more comorbidities. ${ }^{8,9}$ These multimorbidities were associated with higher health care costs, ED visits, mortality, and readmissions. ${ }^{10-12}$

Readmission serves as one important indicator measuring the quality and utilization of expensive inpatient care that adds to total health care costs. In 2012, due to a trend of increasing inpatient total charges for 30-day COPDrelated readmissions, ${ }^{13}$ the Centers for Medicare and Medicaid services (CMS) initiated the Hospital Readmissions Reduction Program (HRRP). By penalizing hospitals for excessive 30-day readmission rates for six disease- or procedure-specific indicators, this program aimed to decrease excessive medical costs. One of these six indicators is COPD.

Reducing 30-day COPD-related readmission, however, can be challenging, especially for patients with comorbidities, because no existing guidance is applicable. Even though comorbidities are important factors in predicting the risk of 30-day readmission, ${ }^{10,14}$ the management and the medical intervention in COPD patients with comorbidities is not clearly established worldwide. ${ }^{15}$ Research has shown that COPD comorbid with other diseases, such as heart disease, ${ }^{16,17}$ depression ${ }^{16,17}$ and diabetes, ${ }^{18}$ could increase the risk of 30-day readmission. However, none of these studies were representative, and none took multimorbidity into consideration. ${ }^{16-18}$ Two studies analyzed only one state within the US (one in Florida and the other in California), ${ }^{16,17}$ and the other study only used data from private health insurance plans. ${ }^{18}$ Furthermore, they solely considered the impact of one comorbidity and did not evaluate how the increasing number of comorbidities would affect 30-day readmission. Given the importance of disease complexity in COPD-related 30-day readmission, evaluating and comparing the impact of multimorbidity on 30-day readmission is substantial. However, only limited studies to date have examined the risk of 30-day readmission between COPD patients with and without additional comorbidities. We hypothesized that COPD patients who had comorbidities would show higher 30-day readmission risk compared to those who did not present comorbidities, and the risk would raise as the number of comorbidities increased. The primary aim of this study is to test this hypothesis.

In addition, this study has two secondary aims. First, this study tests the gender effect. Prior research reported that male and female patients were different in terms of COPDrelated 30-day readmission. ${ }^{7,11,19}$ Males were more likely to be readmitted, ${ }^{19,20}$ whereas females had fewer comorbidities and might be undertreated for COPD, itself. ${ }^{21}$ Although gender plays an important role in COPD readmission, few studies have navigated how gender might affect the COPD-related 30-day readmission rate among patients with and without additional comorbidities. We hypothesized that both male and female COPD patients with comorbidities were more likely to be readmitted, compared to male patients without comorbidities. Second, this study differentiates the effects between having public (Medicare and Medicaid) or private and other health insurance. Evidence has shown that COPD patients enrolled in public insurance programs had a higher risk of being readmitted, because Medicaid enrollees tend to represent lower socioeconomic groups (cite), while Medicare beneficiaries represent an older population, ${ }^{19}$ and both groups generally have more complex health needs. We hypothesized that, among patients with public insurance, those with comorbidities had higher risks of being readmitted within 30 days, as compared to COPD patients without comorbidities.

\section{Methods}

\section{Study Population}

We used all-payer admissions in the Healthcare Cost and Utilization Project (HCUP) of the State Inpatient Databases (SID) in this multistate retrospective cohort study. HCUP SID are state-specific files that contain all inpatient admission records in participating states, encompassing more than $95 \%$ of all US hospital discharges. The SID are well suited for research that requires enumeration of hospitals and discharges within geographic areas or states. $^{22}$ Because of this feature, it is one of the strengths of using SID to evaluate the 30-day readmission risks among COPD patients.

Our study population consisted of COPD patients from nine states, including Arkansas, Florida, Iowa, Massachusetts, New Jersey, Nebraska, New York, Utah, and Washington. Patients were aged 40 years or older ${ }^{23}$ and had been admitted into hospitals between January 1, 2013, to December 31, 2013. We followed the Agency for Healthcare Research and Quality 
(AHRQ) guidance and software in developing an index to identify COPD patients - those who had International Classification of Diseases, Ninth Revision, Clinical Modification (ICD-9-CM) codes 491.0, 491.1, 491.20, 491.21, 491.22, 491.8, 491.9 [chronic bronchitis], 492.0, 492.8 [emphysema], 494.0, 494.1 [bronchiectasis] or 496.xx [COPD, unspecified]. ${ }^{24}$ Even though bronchiectasis differs from COPD clinically, we still include bronchiectasis as part of COPD definition. Bronchiectasis and COPD clinically can be overlapped, and sometimes bronchiectasis can be misreported as COPD. ${ }^{25}$ According to the definition of Prevention Quality Indicator 05 (PQI 05) of COPD, provided by AHRQ, bronchiectasis is included. ${ }^{26}$ We chose their first admission within 2013 as the index date and excluded those who had asthma, who had planned readmissions, who were discharged against medical advice, who reported a same-day referral at the same hospitals for the same principal diagnosis, and who were transferred to short-term hospitals.

This study was approved by Virginia Commonwealth University's (VCU) institutional review board (IRB). Informed consent was waived by the VCU IRB, because we used administrative data collected in 2013 in the form of a limited data set. Patients' individual IDs were deidentified in State Inpatient Databases (SID) to ensure data confidentiality. This study was carried out in accordance with the principles of the Declaration of Helsinki.

\section{Outcomes}

The main outcome was COPD-related 30-day readmission. We defined COPD-related 30-day readmission as patients who had unplanned readmission within 30 days after discharge and had COPD as their principal diagnosis.

\section{Covariates}

Covariates were extracted from three data sources: HCUP SID, Area Health Resources Files (AHRF) and the American Hospital Association (AHA) annual survey database. The major independent variable was a set of comorbidities. We used Elixhauser methodology to develop comorbidity measures. The AHRQ provides Elixhauser Comorbidity software to identify major chronic comorbidities for risk adjustment of the HCUP SID. The software creates 29 binary variables, excluding complications, ${ }^{27,28}$ based on the International Classification of Diseases (ICD) 9th revision, clinical modification (ICD-9-CM). Congestive heart failure, diabetes, depression, and peripheral vascular disease that frequently occur in COPD patients are among those identified. ${ }^{27,29} \mathrm{We}$ used administrative data that were compiled after the primary diagnoses were confirmed. Thus, we excluded all secondary COPD diagnosis as we wanted to assess the effects of other comorbidities on patients with primary COPD diagnosis. Using these 29 comorbidity indicators, we created 4 mutually exclusive comorbidity indicator variables. ${ }^{30}$ First, we identified patients who had only a COPD diagnosis, meaning they did not have any comorbidities. Second, we then categorized the rest of COPD patients into having one, two to three, and four or more comorbidities.

Previous studies suggested that many factors could influence COPD-related 30-day readmissions. In this study, we categorized them into three levels: individual patient, hospital, and geographic. Individual-level factors, obtained from HCUP SID, included gender, race, age and types of primary insurance payer. ${ }^{7,16,31}$ Hospital-level factors included number of beds; teaching hospital; residency training approval hospital; hospital location and Medicare Accountable Care Organization (ACO) types: Pioneer or Medicare Shared Savings Program (MSSP); ${ }^{32,33}$ information collected from the AHA Annual Survey and the level of implementation of health information technology, collected from the Health Information and Management System Society. Geographic level factors consisted of median household income (reported by quartiles of the patient's ZIP code and acquired from AHRF), Herfindahl-Hirschman index (defined as the sum of square market shares of inpatient days measured in a county, ${ }^{34}$ which was obtained from AHA survey), and state-fixed effects. ${ }^{35,36}$

\section{Statistical Analysis}

To access the impact of different levels of factors on COPDrelated 30-day readmission, we built our models cumulatively. First, we built up a crude model where only comorbidities were included (model 1). Second, we considered outcomes were confounded by individual patient characteristics and added them into the regression model (model 2). Third, we then added hospital factors into model 2 in creating model 3 to examine how hospital-level factors, after controlling individual factors, would affect outcome estimates. Finally, we added geographic level factors into model 3 in creating model 4 . While we were not able to distinguish effects between individual factors and other two (hospital and geographic factors), by creating models aggregately, we could evaluate what level of factors could best explain unobservable variations.

We estimated hazards of 30-day readmission, using Cox regression to compute crude and adjusted hazard ratios (HRs) with 95\% CIs for the individual outcomes associated with four comorbidity groups, and with COPD patients 
having no comorbidities as the reference group. First, without controlling any other covariates but comorbidities, we estimated the crude hazard ratio of 30-day readmission.

Second, we compute adjusted hazard ratios considering outcomes first confounded only by individual characteristics. Then, we added hospital- and geographic level factors, cumulatively. Finally, we stratified by gender and primary insurance payer types to examine their effects, respectively. With the assumption underline Cox regression, we assume the hazard functions for different levels of comorbidities are proportional for all time points.

\section{Results}

\section{Basic Characteristics}

The final analyzable sample, following the sample selection process showed in Figure 1, contains 9982 COPD-diagnosed patients. Among them, 824 patients have only a COPD diagnosis, 1839 patients have COPD and one comorbidity diagnosis, 4606 patients have two to three comorbidities, and 2713 patients have four or more comorbidities.

As shown in Table 1, on individual-level characteristics, comorbidity groups distribute differently among COPD patients according to gender, age, race, and insurance types. Males are less likely to be diagnosed with comorbidities compared to females. However, as the number of comorbidities increases, the percentage of diagnosed males decreases (from $49.39 \%$ with zero comorbidity to $42.39 \%$ with four or more comorbidities, $\mathrm{p}<0.0001$ ); in contrast, the proportion of females increases (from 50.61\% to $57.61 \%, \mathrm{p}<0.0001)$. Regarding age, elderly people are more likely to have more complex comorbidities. The average age for patients who have no comorbidity is 65.77

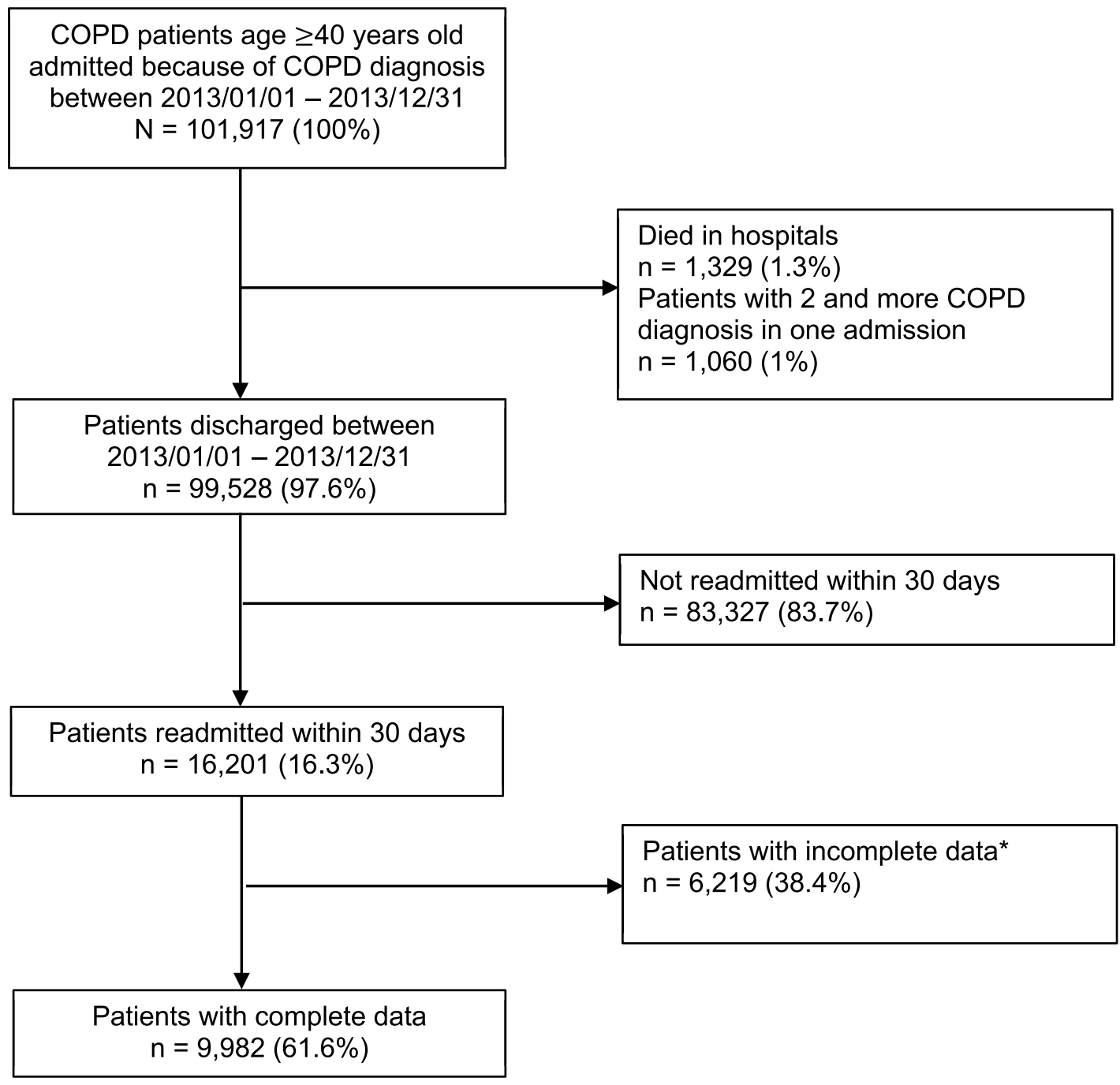

Figure I Sample selection process.

Note: *Incomplete data imply those who had missing value in controlling variables. 
Table I Sample Characteristics, by Comorbidity Categorizations, January 2013-December 2013

\begin{tabular}{|c|c|c|c|c|c|c|c|c|c|}
\hline \multirow[t]{3}{*}{ Comorbidities } & \multicolumn{8}{|c|}{ COPD } & \\
\hline & \multicolumn{2}{|l|}{ Non } & \multicolumn{2}{|l|}{$\mathbf{I}$} & \multicolumn{2}{|l|}{2 to 3} & \multicolumn{2}{|c|}{4 or More } & \multirow[b]{2}{*}{$p$} \\
\hline & $\mathbf{N}$ & $\%$ & $\mathbf{N}$ & $\%$ & $\mathbf{N}$ & $\%$ & $\mathbf{N}$ & $\%$ & \\
\hline All & 824 & 100 & 1839 & 100 & 4606 & 100 & 2713 & 100 & \\
\hline \multicolumn{10}{|l|}{ Individual factors } \\
\hline \multicolumn{10}{|l|}{ Gender } \\
\hline Male & 407 & 49.39 & 845 & 45.95 & 2051 & 44.53 & 1150 & 42.39 & $<0.0001$ \\
\hline Female & 417 & 50.61 & 994 & 54.05 & 2555 & 55.47 & 1563 & 57.61 & \\
\hline Age $^{a}$ (in years) & 65.77 & 11.55 & 67.82 & $\mid 1.41$ & 69.34 & 11.41 & 71 & 11.61 & $<0.0001$ \\
\hline \multicolumn{10}{|l|}{ Race } \\
\hline White & 722 & 87.62 & $154 \mid$ & 83.8 & 3678 & 79.85 & 2119 & 78.11 & $<0.0001$ \\
\hline Black & 32 & 3.88 & 139 & 7.56 & 452 & 9.81 & 313 & 11.54 & \\
\hline Hispanic & 47 & 5.7 & 110 & 5.98 & 343 & 7.45 & 203 & 7.48 & \\
\hline Other & 23 & 2.79 & 49 & 2.66 & 133 & 2.89 & 78 & 2.88 & \\
\hline \multicolumn{10}{|l|}{ Insurance types } \\
\hline Public & 621 & 75.36 & 1505 & 81.84 & 4042 & 87.76 & 2468 & 90.97 & $<0.0001$ \\
\hline Private & 203 & 24.64 & 334 & 18.16 & 564 & 12.24 & 245 & 9.03 & \\
\hline \multicolumn{10}{|l|}{ Hospital factors } \\
\hline \multicolumn{10}{|l|}{ Bed size } \\
\hline 6-199 beds & 320 & 38.83 & 650 & 35.35 & 1412 & 30.66 & 744 & 27.42 & $<0.0001$ \\
\hline 200-349 beds & 270 & 32.77 & 635 & 34.53 & 1760 & 38.21 & 1062 & 39.14 & \\
\hline 500 or more beds & 234 & 28.4 & 554 & 30.13 & 1434 & 31.13 & 907 & 33.43 & \\
\hline \multicolumn{10}{|l|}{ Teaching hospital } \\
\hline Yes & 137 & 16.63 & 345 & 18.76 & 930 & 20.19 & 615 & 22.67 & 0.0013 \\
\hline No & 687 & 83.37 & 1494 & 81.24 & 3676 & 79.81 & 2098 & 77.33 & \\
\hline \multicolumn{10}{|l|}{ Residency training hospital } \\
\hline Yes & 305 & 37.01 & 720 & 39.15 & 1943 & 42.18 & 1230 & 45.34 & $<0.0001$ \\
\hline No & 519 & 62.99 & 1119 & 60.85 & 2663 & 57.82 & 1483 & 54.66 & \\
\hline \multicolumn{10}{|l|}{ Rural hospital } \\
\hline Yes & 117 & 14.2 & 234 & 12.72 & 501 & 10.88 & 251 & 9.25 & 0.0001 \\
\hline No & 707 & 85.8 & 1605 & 87.28 & 4105 & 89.12 & 2462 & 90.75 & \\
\hline \multicolumn{10}{|l|}{ Basic health IT } \\
\hline No & 8 & 0.97 & 22 & 1.2 & 38 & 0.83 & 25 & 0.92 & 0.573 \\
\hline Partially & 329 & 39.93 & 742 & 40.35 & 1857 & 40.32 & 1100 & 40.55 & \\
\hline Fully & 487 & 59.1 & 1075 & 58.46 & 2711 & 58.86 & 1588 & 58.53 & \\
\hline \multicolumn{10}{|l|}{ Geographic factors } \\
\hline \multicolumn{10}{|l|}{ ACO types } \\
\hline \multicolumn{10}{|l|}{ Pioneer } \\
\hline Yes & 39 & 4.73 & 102 & 5.55 & 281 & 6.1 & 202 & 7.45 & 0.004 \\
\hline No & 785 & 95.27 & 1737 & 94.45 & 4325 & 93.9 & 2511 & 92.55 & \\
\hline \multicolumn{10}{|l|}{ MSSP } \\
\hline Yes & 109 & 13.23 & 317 & 17.24 & 868 & 18.84 & 545 & 20.09 & 0.0001 \\
\hline No & 715 & 86.77 & 1522 & 82.76 & 3738 & 81.16 & 2168 & 79.91 & \\
\hline Median income & & & & & & & & & \\
\hline Ist & 259 & 31.43 & 583 & 31.7 & 1486 & 32.26 & 908 & 33.47 & 0.0572 \\
\hline 2nd & 224 & 27.18 & 517 & 28.11 & 1314 & 28.53 & 743 & 27.39 & \\
\hline
\end{tabular}


Table I (Continued).

\begin{tabular}{|c|c|c|c|c|c|c|c|c|c|}
\hline \multirow[t]{3}{*}{ Comorbidities } & \multicolumn{8}{|c|}{ COPD } & \\
\hline & \multicolumn{2}{|c|}{ Non } & \multicolumn{2}{|l|}{ I } & \multicolumn{2}{|c|}{2 to 3} & \multicolumn{2}{|c|}{4 or More } & \\
\hline & $\mathbf{N}$ & $\%$ & $\mathbf{N}$ & $\%$ & $\mathbf{N}$ & $\%$ & $\mathbf{N}$ & $\%$ & $p$ \\
\hline All & 824 & 100 & 1839 & 100 & 4606 & 100 & 2713 & 100 & \\
\hline 3 rd & 219 & 26.58 & 443 & 24.09 & 1060 & 23.01 & 620 & 22.85 & \\
\hline 4th & 122 & $14.8 \mid$ & 296 & 16.1 & 746 & 16.2 & 442 & 16.29 & \\
\hline $\mathbf{H H} \mathbf{I}^{\mathbf{a}}$ & 0.51 & 0.29 & 0.48 & 0.28 & 0.47 & 0.27 & 0.44 & 0.26 & $<0.0001$ \\
\hline
\end{tabular}

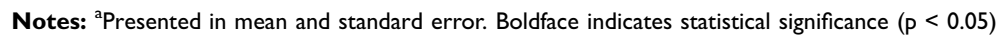

Abbreviation: ACO, Accountable Care Organization.

years, and increases to 71 for those who have four or more comorbidities $(\mathrm{p}<0.0001)$. The majority of these patients are white $(>78 \%)$, and more than $75 \%$ have public insurance (Medicare or Medicaid). Public insurance enrollees tend to have more complex conditions ( $75 \%$ without comorbidity compared to $90.7 \%$ with four or more comorbidities). On the other hand, patients with private or other types of health insurance tend to have fewer comorbidities.

In terms of hospital-level characteristics, COPD patients who have more comorbidities on average stay longer in hospitals (3.78 days without comorbidity compared to 5.27 with four or more comorbidities, $\mathrm{p}<0.0001)$. Additionally, urban and teaching hospitals are more likely to admit patients with more comorbidities.

Regarding geographic level characteristics, as the number of comorbidities increases, the proportion of Medicare Accountable Care Organization (ACO) enrollees increases, and the health care markets become less concentrated.

\section{Risk of 30-Day Readmission}

Table 2 shows the crude and adjusted hazard ratio (HR) of 30-day readmission among comorbid COPD patients. Model 1 presents the crude HR, showing that, although COPD patients without comorbidities have a slightly lower risk of readmission $(\mathrm{HR}=0.91)$, the risk of 30day readmission between COPD patients with and without comorbidities does not differ significantly ( $\mathrm{p}$ value from 0.06 to 0.1$)$.

When taking into account individual-level factors (model 2), the hazard of 30-day readmission differs between COPD patients without comorbidities and those who have comorbidities. Compared to COPD patients without comorbidities, those COPD patients with one $(\mathrm{aHR}=0.84,95 \% \mathrm{CI}: 0.72-0.96, \mathrm{p}=0.013)$ or two to three $(\mathrm{aHR}=0.87,95 \%$ CI: 0.76-0.98, $\mathrm{p}=0.026)$ comorbidities have significantly lower risks of being readmitted within 30 days. For COPD patients have four or more comorbidities $(\mathrm{aHR}=0.87,95 \% \mathrm{CI}$ : 0.76-1.00, $\mathrm{p}=$ 0.05 ), while the 30 -day readmission risk is also lower as compared to those who did not have comorbidities, this risk reduction is not statistically significant. Additionally, these effects remain consistent when we further add hospital- and geographic level factors (models 3 and 4), implying that our basic model could sufficiently explain the majority of possible variations. We observe a slight dose response that, as the number of comorbidities grows, the risk of being readmitted increases from 0.84 to 0.87 .

We also observe that the risk of 30-day readmission differs by gender in which females have lower 30-day readmission risks $(\mathrm{aHR}=0.91,95 \% \mathrm{CI}: 0.85-0.98)$ than males. In addition, we find the median income among different geographic areas could influence 30-day readmission risk. Although only patients living in areas with the highest median income report a significantly lower risk of 30-day readmission ( $\mathrm{aHR}=0.89,95 \% \mathrm{CI}$ : 0.80-1.00, $\mathrm{p}=0.041$, we still observe that the risk of being readmitted decreases as the median income increases.

\section{Stratified Analysis}

We conducted stratified analyses to test two secondary hypotheses. First, we evaluate the gender effect. As shown in Table 2, the 30-day readmission risk performs differently between males and females. We conducted a stratified analysis to evaluate the gender effect (Table 3 ). Table 3 shows that females with comorbidities, compared to those without, are less likely to be readmitted within 30 days (aHR from 0.80 to 0.85 ). A similar trend also appears among males. Interestingly, regardless of how many comorbidities they have, we observed that males with comorbidities, as 
Table 2 COPD Comorbidity Categorization and 30-Day Readmission Risk, by Different Levels of Controlling Variables

\begin{tabular}{|c|c|c|c|c|c|c|c|c|c|c|c|c|}
\hline \multicolumn{13}{|l|}{ 30-Day Readmission } \\
\hline \multirow[t]{3}{*}{ Model } & \multicolumn{3}{|l|}{1} & \multicolumn{3}{|l|}{2} & \multicolumn{3}{|l|}{3} & \multicolumn{3}{|l|}{4} \\
\hline & \multicolumn{3}{|c|}{ Crude } & \multicolumn{3}{|c|}{ I + Individual Factors } & \multicolumn{3}{|c|}{$2+$ Hospital Factors } & \multicolumn{3}{|c|}{3 + Geographic Factors } \\
\hline & $\mathbf{H R}^{\mathbf{a}}$ & $95 \% \mathrm{Cl}$ & $p$ & $\mathrm{aHR}^{\mathrm{b}}$ & $95 \% \mathrm{Cl}$ & $p$ & $\mathrm{aHR}^{\mathrm{b}}$ & $95 \% \mathrm{Cl}$ & $p$ & $\mathrm{aHR}^{\mathrm{b}}$ & $95 \% \mathrm{Cl}$ & $p$ \\
\hline COPD & 1 & Ref. & & 1 & Ref. & & 1 & Ref. & & 1 & & \\
\hline COPD + 1 & 0.91 & $(0.81,1.02)$ & 0.09 & $0.84 *$ & $(0.72,0.96)$ & 0.013 & $0.84 *$ & $(0.73,0.96)$ & 0.014 & $0.83^{*}$ & $(0.72,0.96)$ & 0.011 \\
\hline COPD + 2-3 & 0.91 & $(0.82,1.01)$ & 0.064 & $0.87^{*}$ & $(0.76,0.98)$ & 0.026 & $0.87 *$ & $(0.77,0.99)$ & 0.032 & $0.87 *$ & $(0.76,0.99)$ & 0.03 \\
\hline COPD +4 and more & 0.91 & $(0.82,1.02)$ & 0.096 & 0.87 & $(0.76,1.00)$ & 0.050 & 0.88 & $(0.77,1.01)$ & 0.068 & 0.88 & $(0.77,1.01)$ & 0.059 \\
\hline \multicolumn{13}{|l|}{ Individual factors } \\
\hline \multicolumn{13}{|l|}{ Gender } \\
\hline Male & & & & 1 & Ref. & & 1 & Ref. & & 1 & Ref. & \\
\hline Female & & & & $0.91 *$ & $(0.85,0.98)$ & 0.010 & $0.91 *$ & $(0.85,0.98)$ & 0.013 & $0.92^{*}$ & $(0.85,0.98)$ & 0.014 \\
\hline Age & & & & 1 & $(1.00,1.00)$ & 0.488 & 1 & $(1.00,1.00)$ & 0.421 & 1 & $(1.00,1.00)$ & 0.336 \\
\hline \multicolumn{13}{|l|}{ Race } \\
\hline White & & & & 1 & Ref. & & 1 & Ref. & & 1 & Ref. & \\
\hline Black & & & & $0.87^{*}$ & $(0.76,0.98)$ & 0.027 & $0.87^{*}$ & $(0.77,0.99)$ & 0.036 & $0.86^{*}$ & $(0.75,0.98)$ & 0.023 \\
\hline Hispanic & & & & 0.96 & $(0.83,1.10)$ & 0.535 & 0.96 & $(0.84,1.11)$ & 0.615 & 0.96 & $(0.83,1.11)$ & 0.588 \\
\hline Other & & & & 0.94 & $(0.76,1.17)$ & 0.596 & 0.95 & $(0.77,1.17)$ & 0.616 & 0.95 & $(0.77,1.18)$ & 0.653 \\
\hline \multicolumn{13}{|l|}{ Insurance types } \\
\hline Public & & & & 1 & & & 1 & & & 1 & & \\
\hline Private and others & & & & 0.96 & $(0.86,1.07)$ & 0.436 & 0.96 & $(0.86,1.07)$ & 0.487 & 0.98 & $(0.88,1.09)$ & 0.654 \\
\hline \multicolumn{13}{|l|}{ Hospital level } \\
\hline \multicolumn{13}{|l|}{ Bed size } \\
\hline $6-199$ beds & & & & & & & 1 & Ref. & & 1 & Ref. & \\
\hline $200-349$ beds & & & & & & & 0.98 & $(0.89,1.07)$ & 0.629 & 1 & $(0.92,1.10)$ & 0.92 \\
\hline 500 or more beds & & & & & & & 0.98 & $(0.87,1.11)$ & 0.794 & 1.01 & $(0.89,1.15)$ & 0.849 \\
\hline \multicolumn{13}{|l|}{ Teaching hospital } \\
\hline No & & & & & & & 1 & Ref. & & 1 & Ref. & \\
\hline Yes & & & & & & & 1.03 & $(0.94,1.14)$ & 0.483 & 1.03 & $(0.94,1.14)$ & 0.505 \\
\hline \multicolumn{13}{|c|}{ Residency training hospital } \\
\hline No & & & & & & & 1 & Ref. & & 1 & Ref. & \\
\hline Yes & & & & & & & 0.94 & $(0.84,1.05)$ & 0.271 & 0.93 & $(0.83,1.05)$ & 0.23 \\
\hline \multicolumn{13}{|l|}{ Rural hospital } \\
\hline No & & & & & & & 1 & Ref. & & 1 & Ref. & 0.424 \\
\hline Yes & & & & & & & 1.1 & $(0.98,1.23)$ & 0.111 & 1.05 & $(0.93,1.18)$ & 0.427 \\
\hline \multicolumn{13}{|l|}{ Basic health IT } \\
\hline No & & & & & & & 1 & & & 1 & & \\
\hline Partially & & & & & & & 1.15 & $(0.79,1.70)$ & 0.464 & 1.09 & $(0.74,1.61)$ & 0.65 \\
\hline Fully & & & & & & & 1.14 & $(0.78,1.67)$ & 0.507 & 1.06 & $(0.72,1.56)$ & 0.774 \\
\hline ACO types & & & & & & & & & & & & \\
\hline Pioneer & & & & & & & & & & & & \\
\hline No & & & & & & & 1 & & & 1 & Ref. & \\
\hline Yes & & & & & & & $0.81 *$ & $(0.69,0.95)$ & 0.010 & 0.85 & $(0.72,1.01)$ & 0.069 \\
\hline MSSP & & & & & & & & & & & & \\
\hline No & & & & & & & 1 & Ref. & & 1 & Ref. & \\
\hline Yes & & & & & & & 1.01 & $(0.92,1.11)$ & 0.846 & 0.98 & $(0.88,1.08)$ & 0.675 \\
\hline
\end{tabular}

(Continued) 
Table 2 (Continued).

\begin{tabular}{|c|c|c|c|c|c|c|c|c|c|c|c|c|}
\hline \multicolumn{13}{|c|}{ 30-Day Readmission } \\
\hline \multirow[t]{3}{*}{ Model } & \multicolumn{3}{|l|}{1} & \multicolumn{3}{|l|}{2} & \multicolumn{3}{|l|}{3} & \multicolumn{3}{|l|}{4} \\
\hline & \multicolumn{3}{|c|}{ Crude } & \multicolumn{3}{|c|}{ I + Individual Factors } & \multicolumn{3}{|c|}{$2+$ Hospital Factors } & \multicolumn{3}{|c|}{3 + Geographic Factors } \\
\hline & $H^{a}{ }^{a}$ & $95 \% \mathrm{Cl}$ & $p$ & $a H R^{b}$ & $95 \% \mathrm{Cl}$ & $p$ & $a H R^{b}$ & $95 \% \mathrm{Cl}$ & $p$ & $\mathrm{aHR}^{\mathrm{b}}$ & $95 \% \mathrm{Cl}$ & $p$ \\
\hline \multicolumn{13}{|c|}{ Geographic level } \\
\hline $\begin{array}{l}\text { Medi } \\
\text { Ist }\end{array}$ & & & & & & & & & & 1 & Ref. & \\
\hline 2nd & & & & & & & & & & 0.97 & $(0.88,1.06)$ & 0.495 \\
\hline $3 r d$ & & & & & & & & & & 0.91 & $(0.83,1.01)$ & 0.071 \\
\hline 4 th & & & & & & & & & & $0.89 *$ & $(0.80,1.00)$ & 0.042 \\
\hline HHI & & & & & & & & & & 1.07 & $(0.90,1.26)$ & 0.438 \\
\hline \multicolumn{13}{|c|}{ State-fixed effects } \\
\hline AR & & & & & & & & & & 1 & Ref. & \\
\hline $\mathrm{FL}$ & & & & & & & & & & 1.09 & $(0.94,1.25)$ & 0.252 \\
\hline IA & & & & & & & & & & $1.4 I^{*}$ & $(1.19,1.68)$ & 0 \\
\hline MA & & & & & & & & & & 1.03 & $(0.87,1.22)$ & 0.747 \\
\hline NY & & & & & & & & & & 1.08 & $(0.94,1.25)$ & 0.269 \\
\hline UT & & & & & & & & & & 0.5 & $(0.13,2.03)$ & 0.336 \\
\hline WA & & & & & & & & & & 1.23 & $(0.98,1.53)$ & 0.072 \\
\hline
\end{tabular}

Notes: ${ }^{*} \mathrm{p}$ value $<0.05$; ${ }^{\mathrm{a} H R}$, crude hazard ratio. ${ }^{\mathrm{b}}$ aHR, adjusted hazard ratio.

Abbreviations: AR, Arkansas; FL, Florida; IA, lowa; MA, Massachusetts; NY, New York; UT, Utah; WA, Washington.

compared to females within the same comorbidity group, seem to have a higher chance of being readmitted within 30 days.

Second, we also assess the impact of health insurance on 30-day readmission stratified by public (Medicare and Medicaid), private, and other health insurance type (shown in Table 3. COPD patients with Medicare or Medicaid coverage, if having comorbidities, are less likely to be readmitted within 30 days (aHR from 0.79 to 0.84 )). This result differs from what we expected. Regarding private and other health insurance, however, we observe that the number of comorbidities does not affect 30-day readmission risks, which is in accordance with our hypothesis.

\section{Discussion}

After the first discharge in 2013, this study showed that COPD patients who had comorbidities were less likely to be readmitted within 30 days, compared to those who had no comorbidities. However, we observed a small incremental effect showing that the more comorbidities patients had, the higher the risk of 30-day readmission they would experience, which echoes past findings. ${ }^{37}$ The stratified analysis indicated that gender and primary insurance payer effects existed. Females who had comorbidities, compared to those who had no comorbidities, had a lower 30-day readmission risk. In comparison, males had a higher 30-day readmission risk than females, regardless of the number of comorbidities they had. Comorbid patients with public insurance, compared to those who had no comorbidities, had a lower 30-day readmission risk. In addition, as the number of comorbidities grew, the risk of readmission for those patients increased slightly.

Though our results might contradict previous findings, ${ }^{20}$ they are aligned with some existing evidence. First, as shown in Figure 1, we found that 16,201 patients $(16.3 \%)$ were readmitted within 30 days, which concurred with past findings of $19.2 \%{ }^{19}$ Moreover, the distribution of comorbidity categories was also similar to previous results. ${ }^{30}$ Second, by using SID and Elixhauser comorbidities variables, previous studies have shown that among comorbid COPD patients - including congestive heart failure, lung cancer, weight loss, deficiency anemias, hypertension, diabetes, hypothyroidism, lymphoma, alcohol abuse, obesity, chronic kidney disease and obstructive sleep apnea - the risk of 30-day all-cause or COPD-related readmission did not differ from patients without comorbidities. $^{13,16,17}$ One possible explanation is that a primary COPD diagnosis among comorbid patients could motivate them to modify their health behaviors by trying to increase physical activities and monitoring their diet. $^{38}$ Furthermore, COPD patients with comorbidities often consulted family physicians more, which may in 
Table 3 COPD Comorbidity Categorization and 30-Day Readmission Risk, Stratified by Gender and Insurance Types

\begin{tabular}{|c|c|c|c|c|c|c|c|c|c|c|c|c|}
\hline & \multicolumn{12}{|c|}{ 30-Day Readmission ${ }^{a}$} \\
\hline & \multicolumn{3}{|c|}{ Female } & \multicolumn{3}{|l|}{ Male } & \multicolumn{3}{|c|}{ Public $^{\text {b }}$} & \multicolumn{3}{|c|}{ Private and Other ${ }^{b}$} \\
\hline & $\mathbf{a H R}$ & $95 \% \mathrm{Cl}$ & $p$ & $\mathbf{a H R}^{\mathbf{c}}$ & $95 \% \mathrm{Cl}$ & $p$ & $\mathbf{a H R}^{\mathbf{c}}$ & $95 \% \mathrm{Cl}$ & $\mathbf{p}$ & $\mathrm{aHR}^{\mathrm{c}}$ & $95 \% \mathrm{Cl}$ & $\mathbf{p}$ \\
\hline COPD & I & Ref. & & 1 & Ref. & & I & Ref. & & 1 & Ref. & \\
\hline COPD + 1 & $0.79 *$ & $(0.65,0.97)$ & 0.024 & 0.89 & $(0.72,1.09)$ & $0.24 I$ & $0.79 * *$ & $(0.67,0.93)$ & 0.004 & 1 & $(0.73,1.36)$ & 0.986 \\
\hline COPD + 2-3 & 0.85 & $(0.71,1.01)$ & 0.069 & 0.9 & $(0.75,1.08)$ & 0.269 & $0.84^{*}$ & $(0.74,0.98)$ & 0.025 & 0.91 & $(0.68,1.21)$ & 0.511 \\
\hline COPD +4 and more & 0.86 & $(0.71,1.03)$ & 0.105 & 0.92 & $(0.75,1.12)$ & 0.387 & $0.85^{*}$ & $(0.73,0.99)$ & 0.039 & 0.91 & $(0.65,1.29)$ & 0.61 \\
\hline
\end{tabular}

Notes: $\mathrm{p}$ value $*<0.05, * *<0.01$; ${ }^{\mathrm{T}}$ The model adjusted for individual-, hospital- and geographic level factors; ${ }^{\mathrm{b}}$ Public insurance includes Medicare and Medicaid enrollees; Private and other includes private, self-pay, no charge. 'aHR, adjusted hazard ratio.

turn have resulted in better health outcomes. ${ }^{38}$ Diabetes patients with COPD were more likely to receive preferential care, for example. ${ }^{39}$

Two probable reasons can explain why our findings differ from earlier literature. First, our estimates are more restricted, as compared to other studies which also acquired readmissions from SID. We only consider readmissions after first discharge in 2013, whereas previous studies consider readmissions not only after the first discharge. ${ }^{13,16}$ As a result, more severe patients could have more readmissions. These could lead to an overestimate of the risk of 30-day readmission. Second, it could be due to the difference of study design. Past literature generate estimates from pooling several years of SID together, 2005-2011 or 2009-2014 for example. By pooling several years of data, it could increase statistical power. However, it could also eliminate potential policy impacts - Hospital Readmissions Reduction Program (HRRP) for instance, since neither of these two studies included years as dummy variables. ${ }^{13,16}$

From physicians' perspectives, there may be a greater perceived need for older persons with multimorbidity to reduce readmission, especially since the HRRP was launched in 2012 and began penalizing hospitals with excess COPD readmission as of October 2014. CMS accesses a hospital's performance relative to other hospitals with a similar proportion of patients who are dually eligible for Medicare and full-benefit Medicaid. After calculating the payment adjustment factor and component results for each hospital based on their performance within a certain period, payment reductions are applied to all Medicare feefor-service (FFS) base operating diagnosis-related group (DRG) payments. The payment is capped at $3 \%{ }^{40}$ Physicians had higher incentives in 2013 to reduce 30-day readmissions among comorbid COPD patients. A study analyzing Florida's COPD patients found that COPDrelated 30-day readmissions decreased between 2012 and
2013, from $7.9 \%$ to $7.6 \%$. $^{13}$ Although we did not directly test the effect of HRRP, we thought two reverse directions could explain this result. On the one hand, the penalty could have encouraged hospitals and physicians to start to provide better care for COPD patients. Under pressure from clearly defined penalties, hospitals started to design readmission reduction programs, ${ }^{41}$ and programs bearing a higher risk may increase the likelihood of reducing preventable readmissions. $^{34}$ But, on the other hand, hospitals may lower readmissions by shifting care to other high-resource settings such as observation stays or emergency room (ED) visits rather than delivering higher quality care, because these settings were exempt from the HRRP. ${ }^{20}$

The stratified analysis showed a gender effect. In this study, we found females, compared to males, had a higher likelihood of reporting multimorbidity. Prior research suggested that men were more likely to self-report having COPD. ${ }^{42}$ In addition, we also found that females, compared to males, had a lower 30-day readmission risk. This finding concurred with previous results, showing females had lower 30 -day all-cause readmissions ${ }^{43}$ and fewer ICU admissions due to COPD exacerbation in the previous year. ${ }^{21}$ Males, based on review and retrospective study, in accordance with our estimates, had a higher frequency of readmission. ${ }^{44,45}$ Because females were less likely to self-report COPD and have COPD as a primary diagnosis, when women were readmitted within 30 days their reasons for readmission may not have been caused by COPD.

Additionally, the stratified analysis showed COPD patients with Medicare and Medicaid coverage had a lower risk of 30-day readmission, compared to those with private and other types of insurance. Previous studies have shown that, for patients discharged with hospice services, those who were dual Medicare- and Medicaideligible were less likely to be readmitted within 30 days compared with other insurances. ${ }^{46}$ One possible reason could be the effect of HRRP. Through initiating negative 
repercussions, this program reduces payments to hospitals with excess COPD readmissions. To avoid this, health care providers have been incentivized to provide a better quality of care that might reduce 30-day readmission risk for patients enrolled in Medicare or Medicaid. Although primary data suggest some impact on readmissions, more research is needed to examine the effect of HRRP under an implementation science framework. ${ }^{20,47}$

\section{Limitations}

Our study has several potential limitations. First, this analysis was based on administrative data; hence, coding, sampling errors and hospital misclassifications were possible. Second, the diagnoses of COPD and comorbidities were based on ICD-9-CM codes in the SID data and did not have clinical confirmation. Nevertheless, SID data are accurate and have been used previously for studying readmission risk. ${ }^{30}$ Third, there may also be some potential selection bias, since only patients with complete data were included in our study. However, as we presented in Supplemental Table S1, the covariates of patients with incomplete data were distributed similar to those who had complete data. Fourth, in line with other observational studies, our model might be influenced by unobservable variables. For example, the severity of COPD at the time of admission, the outpatient chronic disease management, and the degree of patients' selfmanagement after discharge. Fifth, we only included 1 year as our observation time, which may affect the statistical power of the analysis. Nevertheless, we included data from nine states of the US, which encompassed an adequate sample population. Sixth, our data were not randomly selected from all US patients with COPD and thus its generalizability may be influenced. Nevertheless, the nine states we chose covered large numbers of patients and different geographic locations, which would tend to increase our estimates' generalizability. In addition, as we provided in Supplemental Table S2, the COPD categorizations distributed similarly between samples that were analyzed and patients who met our excluding criteria. Finally, there might be a selection bias present. However, as shown in Supplemental Table S3, we used Heckman selection model that showed no selection problem.

\section{Conclusion}

In this large, all-payer, population-based retrospective study, we found that, compared with non-comorbid patients, COPD patients with comorbidities had a lower risk of 30-day readmission. Additionally, we also found females and public insurance enrollees had a lower risk of 30-day readmission. In sum, our estimates suggested that COPD patients with comorbidities, being females, and enrolled in public insurance programs were less likely to have unplanned readmission within 30 days of first discharge in 2013. Our findings cannot distinguish whether the lower 30-day readmission risk among COPD patients with multimorbidity could be attributed to better care quality or underdiagnoses and delays in treatment. Future research could encompass more years in order to assess the policy effect and could incorporate outpatient and emergency department data to assess hospitals' strategies to decrease readmissions and patients' adherence to treatments.

\section{Acknowledgment}

We thank Dr Brenda Denzler for helping edit the manuscript.

\section{Author Contributions}

$\mathrm{HX}, \mathrm{AC}$, and SL made significant contributions to the conception and study design, data analyses, and interpretation of results; YD contributed to the data analyses, and manuscript revision; and SL, HX, and AC drafted the article. All authors approved the final manuscript submitted and agreed to be accountable for the work in ensuring the accuracy and integrity.

\section{Funding}

Agency for Healthcare Research and Quality, R01 HS023332.

\section{Disclosure}

The authors declare no competing interests.

\section{References}

1. Singh D, Agusti A, Anzueto A, et al. Global strategy for the diagnosis, management, and prevention of chronic obstructive lung disease: the GOLD science committee report 2019. Eur Respir J. 2019;53 (5):1900164. doi:10.1183/13993003.00164-2019

2. World Health Organization. COPD: definition. Chronic respiratory diseases; 2019. Available from: https://www.who.int/respiratory/copd/ definition/en/. Accessed November 13, 2019. .

3. Smith MC, Wrobel JP. Epidemiology and clinical impact of major comorbidities in patients with COPD. Int J Chron Obstruct Pulmon Dis. 2014;9:871-888. doi:10.2147/COPD

4. Murray CJ, Atkinson C, Bhalla $\mathrm{K}$, et al. The state of US health, 1990-2010: burden of diseases, injuries, and risk factors. JAMA. 2013;310(6):591-608. doi:10.1001/jama.2013.13805

5. Guarascio AJ, Ray SM, Finch CK, Self TH. The clinical and economic burden of chronic obstructive pulmonary disease in the USA. Clinicoecon Outcomes Res. 2013;5:235-245. doi:10.2147/CEOR. S34321

6. Huber MB, Wacker ME, Vogelmeier CF, Leidl R. Excess costs of comorbidities in chronic obstructive pulmonary disease: a systematic review. PLoS One. 2015;10(4):e0123292. doi:10.1371/journal.pone.0123292 
7. Westney G, Foreman MG, Xu J, Henriques King M, Flenaugh E, Rust G. Impact of comorbidities among medicaid enrollees with chronic obstructive pulmonary disease, United States, 2009. Prev Chronic Dis. 2017;14:E31. doi:10.5888/pcd14.160333

8. Yin HL, Yin SQ, Lin QY, Xu Y, Xu HW, Liu T. Prevalence of comorbidities in chronic obstructive pulmonary disease patients: a meta-analysis. Medicine. 2017;96(19):e6836. doi:10.1097/MD.0000 000000006836

9. Anecchino C, Rossi E, Fanizza C, De Rosa M, Tognoni G, Romero M. Prevalence of chronic obstructive pulmonary disease and pattern of comorbidities in a general population. Int $J$ Chron Obstruct Pulmon Dis. 2007;2(4):567-574.

10. Schwab P, Dhamane AD, Hopson SD, et al. Impact of comorbid conditions in COPD patients on health care resource utilization and costs in a predominantly medicare population. Int J Chron Obstruct Pulmon Dis. 2017;12:735-744. doi:10.2147/COPD.S112256

11. Kaszuba E, Odeberg H, Rastam L, Halling A. Impact of heart failure and other comorbidities on mortality in patients with chronic obstructive pulmonary disease: a register-based, prospective cohort study. BMC Fam Pract. 2018;19(1):178. doi:10.1186/s12875-018-0865-8

12. Press VG, Konetzka RT, White SR. Insights about the economic impact of chronic obstructive pulmonary disease readmissions post implementation of the hospital readmission reduction program. Curr Opin Pulm Med. 2018;24(2):138-146. doi:10.1097/MCP.0000000 000000454

13. Jiang X, Xiao H, Segal R, Mobley WC, Park H. Trends in readmission rates, hospital charges, and mortality for patients with chronic obstructive pulmonary disease (COPD) in Florida from 2009 to 2014. Clin Ther. 2018;40(4):613-626.e611. doi:10.1016/j. clinthera.2018.03.006

14. Vogelmeier CF, Criner GJ, Martinez FJ, et al. Global strategy for the diagnosis, management, and prevention of chronic obstructive lung disease 2017 report. GOLD executive summary. Am J Respir Crit Care Med. 2017;195(5):557-582. doi:10.1164/rccm.2017010218PP

15. Hillas G, Perlikos F, Tsiligianni I, Tzanakis N. Managing comorbidities in COPD. Int J Chron Obstruct Pulmon Dis. 2015;10:95-109. doi:10.2147/COPD.S54473

16. Simmering JE, Polgreen LA, Comellas AP, Cavanaugh JE, Polgreen PM. Identifying patients with COPD at high risk of readmission. Chronic Obstr Pulm Dis. 2016;3(4):729. doi:10.15326/ jcopdf.3.4.2016.0136

17. Sharif R, Parekh TM, Pierson KS, Kuo YF, Sharma G. Predictors of early readmission among patients 40 to 64 years of age hospitalized for chronic obstructive pulmonary disease. Ann Am Thorac Soc. 2014;11(5):685-694. doi:10.1513/AnnalsATS.201310-358OC

18. Roberts MH, Clerisme-Beaty E, Kozma CM, Paris A, Slaton T, Mapel DW. A retrospective analysis to identify predictors of COPD-related rehospitalization. BMC Pulm Med. 2016;16(1):68. doi:10.1186/s12890-016-0231-3

19. Jacobs DM, Noyes K, Zhao J, et al. Early hospital readmissions after an acute exacerbation of chronic obstructive pulmonary disease in the nationwide readmissions database. Ann Am Thorac Soc. 2018;15 (7):837-845. doi:10.1513/AnnalsATS.201712-913OC

20. Shah T, Press VG, Huisingh-Scheetz M, White SR. COPD readmissions: addressing COPD in the era of value-based health care. Chest. 2016;150(4):916-926. doi:10.1016/j.chest.2016.05.002

21. Kilic H, Kokturk N, Sari G, Cakir M. Do females behave differently in COPD exacerbation? Int $J$ Chron Obstruct Pulmon Dis. 2015;10:823-830. doi:10.2147/COPD.S78952

22. HCUP State Inpatient Databases (SID). Healthcare cost and utilization project (HCUP); 2013. Available from: www.hcup-us.ahrq.gov/ sidoverview.jsp. Accessed August 7, 2019.

23. Lehrke M, Marx N. Diabetes mellitus and heart failure. Am J Cardiol. 2017;120(1s):S37-S47. doi:10.1016/j.amjcard.2017.05.014
24. Agency for Healthcare Research and Quality. Prevention Quality Indicators technical specifications: version 6; 2016. Available from: https://www.qualityindicators.ahrq.gov/Archive/PQI_TechSpec ICD09_v60.aspx. Accessed August 6, 2019.

25. Chalmers JD. Bronchiectasis and COPD overlap: a case of mistaken identity? Chest. 2017;151(6):1204-1206. doi:10.1016/j.chest.2016. 12.027

26. Agency for Healthcare Research and Quality. Prevention quality indicator 05 (PQI 05) chronic obstructive pulmonary disease (COPD) or asthma in older adults admission rate; 2016. Available from: https://www.qualityindicators.ahrq.gov/Downloads/Modules/ PQI/V60-ICD09/TechSpecs/PQI_05_Chronic_Obstructive_ Pulmonary_Disease_(COPD)_or_Asthma_in_Older_Adults_ Admission_Rate.pdf. Accessed November 26, 2019.

27. Elixhauser A, Steiner C, Harris DR, Coffey RM. Comorbidity measures for use with administrative data. Med Care. 1998;36(1):8-27. doi:10.1097/00005650-199801000-00004

28. Quan H, Sundararajan V, Halfon P, et al. Coding algorithms for defining comorbidities in ICD-9-CM and ICD-10 administrative data. Med Care. 2005;43(11):1130-1139. doi:10.1097/01. mlr.0000182534.19832.83

29. Greulich T, Weist BJD, Koczulla AR, et al. Prevalence of comorbidities in COPD patients by disease severity in a German population. Respir Med. 2017;132:132-138. doi:10.1016/j.rmed.2017.10.007

30. Goto T, Faridi MK, Gibo K, et al. Trends in 30-day readmission rates after COPD hospitalization, 2006-2012. Respir Med. 2017;130:92-97. doi:10.1016/j.rmed.2017.07.058

31. Divo M, Cote C, de Torres JP, et al. Comorbidities and risk of mortality in patients with chronic obstructive pulmonary disease. Am J Respir Crit Care Med. 2012;186(2):155-161. doi:10.1164/rccm.201201-0034OC

32. Bennett KJ, Probst JC, Bullard JC, Crouch E. The importance of rural hospitals: transfers and 30-day readmissions among rural residents and patients presenting at rural hospitals. Popul Health Manag. 2019;22(2):120-126. doi:10.1089/pop.2018.0050

33. Duggal R, Zhang Y, Diana ML. The association between hospital ACO participation and readmission rates. $J$ Healthc Manag. 2018;63 (5):e100-e114. doi:10.1097/JHM-D-16-00045

34. Chukmaitov A, Harless DW, Bazzoli GJ, Muhlestein DB. Preventable hospital admissions and 30-day all-cause readmissions: does hospital participation in accountable care organizations improve quality of care? Am J Med Qual. 2019;34(1):14-22. doi:10.1177/1062860618778786

35. Shrestha PL, Shrestha PA, Vivo RP. Epidemiology of comorbidities in patients with hypertension. Curr Opin Cardiol. 2016;31 (4):376-380. doi:10.1097/HCO.0000000000000298

36. Aggarwal AK, Sujenthiran A, Lewis D, et al. Impact of patient choice and hospital competition on patient outcomes after prostate cancer surgery: a national population-based study. Cancer. 2019;125:1898-1907. doi:10.1002/cncr.31987

37. Roberts CM, Stone RA, Lowe D, Pursey NA, Buckingham RJ. Comorbidities and 90-day outcomes in hospitalized COPD exacerbations. Copd. 2011;8(5):354-361. doi:10.3109/15412555.2011.600362

38. Ansari S, Hosseinzadeh H, Dennis S, Zwar N. Patients' perspectives on the impact of a new COPD diagnosis in the face of multimorbidity: a qualitative study. NPJ Prim Care Respir Med. 2014;24:14036. doi:10.1038/npjpcrm.2014.36

39. Glaser S, Kruger S, Merkel M, Bramlage P, Herth FJ. Chronic obstructive pulmonary disease and diabetes mellitus: a systematic review of the literature. Respiration. 2015;89(3):253-264. doi:10.1159/000369863

40. Centers for Medicare \& Medicaid Services. Reducing chronic obstructive pulmonary disease hospital readmissions. An official American Thoracic Society Workshop ReportMedicare C for Baltimore. MS 7500 SB, USA. Readmissions-Reduction-Program; 2016. Available from: https://www.cms.gov/medicare/medicare-feefor-service-payment/acuteinpatientpps/readmissions-reductionprogram.html. Accessed August 7, 2019. 
41. Press VG, Au DH, Bourbeau J, et al. Reducing chronic obstructive pulmonary disease hospital readmissions. An Official American Thoracic society workshop report. Ann Am Thorac Soc. 2019;16 (2):161-170. doi:10.1513/AnnalsATS.201811-755WS

42. Han MK, Postma D, Mannino DM, et al. Gender and chronic obstructive pulmonary disease: why it matters. Am J Respir Crit Care Med. 2007;176(12):1179-1184. doi:10.1164/rccm.200704-553CC

43. Bashir B, Schneider D, Naglak MC, Churilla TM, Adelsberger M. Evaluation of prediction strategy and care coordination for COPD readmissions. Hosp Pract (1995). 2016;44(3):123-128. doi:10.1080/ 21548331.2016.1210472

44. Cerezo Lajas A, Gutierrez Gonzalez E, Llorente Parrado C, Puente Maestu L, de Miguel-diez J. Readmission due to exacerbation of COPD: associated factors. Lung. 2018;196(2):185-193. doi:10.1007/ s00408-018-0093-y
45. Aryal S, Diaz-Guzman E, Mannino DM. COPD and gender differences: an update. Transl Res. 2013;162(4):208-218. doi:10.1016/j. trs1.2013.04.003

46. Whitney P, Chuang EJ. Relationship between insurance and 30-day readmission rates in patients 65 years and older discharged from an acute care hospital with hospice services. J Hosp Med. 2016;11 (10):688-693. doi:10.1002/jhm.v11.10

47. Villalobos Dintrans P, Bossert TJ, Sherry J, Kruk ME. A synthesis of implementation science frameworks and application to global health gaps. Global Health Res Policy. 2019;4:25. doi:10.1186/s41256-019-0115-1

\section{Publish your work in this journal}

The International Journal of COPD is an international, peer-reviewed journal of therapeutics and pharmacology focusing on concise rapid reporting of clinical studies and reviews in COPD. Special focus is given to the pathophysiological processes underlying the disease, intervention programs, patient focused education, and self management protocols. This journal is indexed on PubMed Central, MedLine and CAS. The manuscript management system is completely online and includes a very quick and fair peer-review system, which is all easy to use. Visit http://www.dovepress.com/testimonials.php to read real quotes from published authors. 